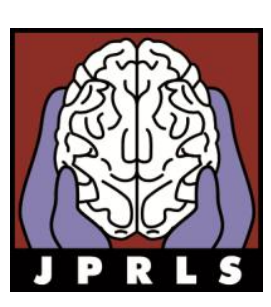

Open Access via www.jprls.org

Journal of Parkinsonism \&

CASE

Restless Legs Syndrome

REPORT

Volume 2

Issue 1 (Apr. 2012)

\title{
Improvement in Central Pontine Myelinosis with IV immunoglobulin
}

\author{
Vesper Fe Marie Llaneza Ramos, ${ }^{1 *} \mathrm{~J}$ Americo Fernandes Filho, ${ }^{1}$ Arun Raj Antony, ${ }^{1} \mathrm{Jason} \mathrm{T}$. \\ Helvey, ${ }^{2}$ John Bertoni ${ }^{1}$ \\ Department of Neurological Sciences, University of Nebraska Medical Center \\ Department of Radiology, University of Nebraska Medical Center
}

\section{INTRODUCTION}

Central pontine myelinolysis (CPM) is a rare, serious demyelinating disease, associated with rapid correction of chronic hyponatremia, usually in the setting of alcoholism and electrolyte disturbance. ${ }^{1} 2$ Magnetic resonance imaging (MRI) is a decisive diagnostic tool for CPM. ${ }^{3}$ The pathogenesis is unknown and there is no established treatment. There have been case reports on the efficacy of intravenous immunoglobulin (IVIG). ${ }^{4} 5$ Postulated mechanisms include reduction of myelinotoxic agents, development of antimyelin antibodies, and promotion of remyelination. ${ }^{4}$

We report a case of CPM associated with chronic alcoholism and hyponatremia, with significant clinical improvement and resolution of MRI changes after treatment with IVIG.

\section{LEVEL OF EVIDENCE}

This report provides Class IV evidence that IVIG $0.4 \mathrm{~g} / \mathrm{kg}$ daily for five days is associated with clinical and radiologic improvement in CPM.

\footnotetext{
* Correspondence to: Vesper Fe Marie Llaneza Ramos, MD, University of Nebraska Medical Center, 982045 Nebraska Medical Center, Omaha, NE 68198-2045. Telephone: (402) 889-2284. Email: vesper.ramos@yahoo.com.
}

\section{CASE REPORT}

A 61-year-old man with a history of chronic alcoholism was admitted in acute ethanol intoxication with a serum ethanol $\sim 300 \mathrm{mg} / \mathrm{dl}$ and sodium 121 $\mathrm{mmol} / \mathrm{L}$. Sodium was corrected to $129 \mathrm{mmol} / \mathrm{L}$ in the first 12 hours; to 132 in the next 4 hours; to 133 the following day; and a slow increase to 140 within 5 days. The patient then became obtunded and dysarthric, and began drooling. Upon transfer to our facility, he was oriented only to person but followed simple commands. He had bilateral ptosis, facial weakness, dysarthria, and hypotonia with quadriparesis (motor strength of $3 / 5$ proximally and $4 / 5$ distally). Reflexes were reduced in the upper extremities and absent in the lower extremities. He had a left extensor plantar response. Initial MRI revealed increased signal on $\mathrm{T} 2$ weighted (T2W) and attenuated inversion recovery (FLAIR), positive diffusion restriction, and decreased $\mathrm{T} 1$ weighted (T1W) signal in the central pontine area, congruent with acute-subacute CPM (Figure 1. A and B.)

He was treated with IVIG $0.4 \mathrm{~g} / \mathrm{kg}$ daily for 5 days. On day 3 , he was more lethargic, with worse ptosis and facial weakness. Strength was $1 / 5$ in the upper extremities and $2 / 5$ in the lower extremities. Repeat MRI showed increased pontine T2W hyperintensity (Figure 2A). IVIG was continued and he gradually improved. By $6^{\text {th }}$ day post treatment, he was deemed fit for discharge, being awake, alert, following two-step commands, with improved facial weakness, no ptosis and had 4/5 strength in all four 

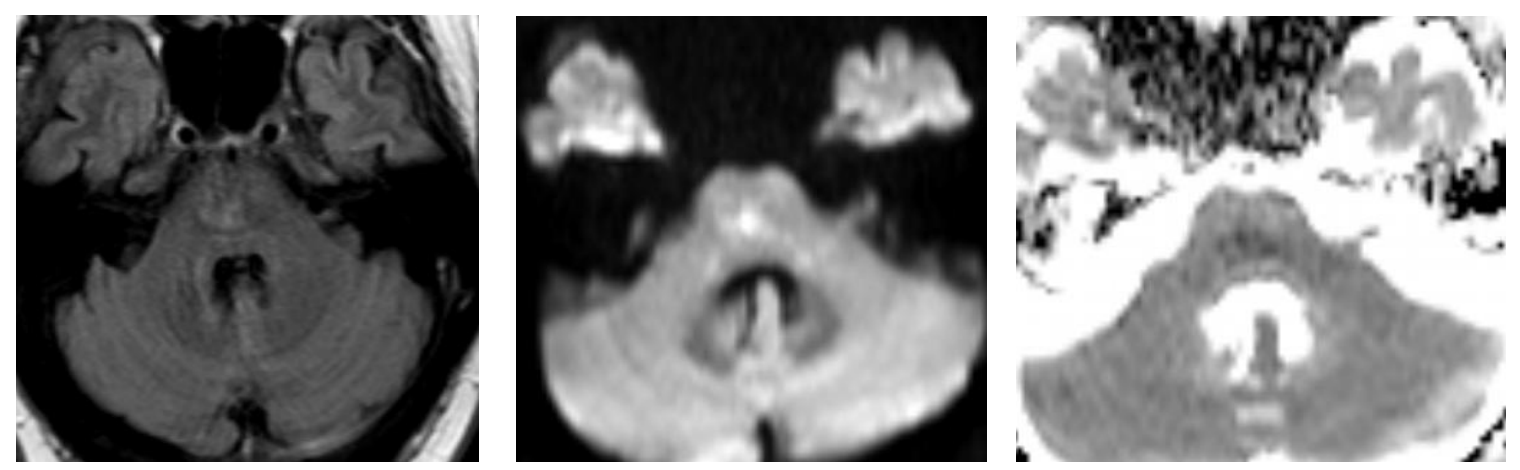

Figure 1. Initial MRI in patient with chronic alcoholism and hyponatremia with flaccid paralysis and mental status change. Left-to-right: T2-weighted fluid attenuated inversion recovery (FLAIR) sequence shows hyperintensity in central pons; Diffusion and apparent diffusion coefficient sequences show diffusion restriction in the central pons.
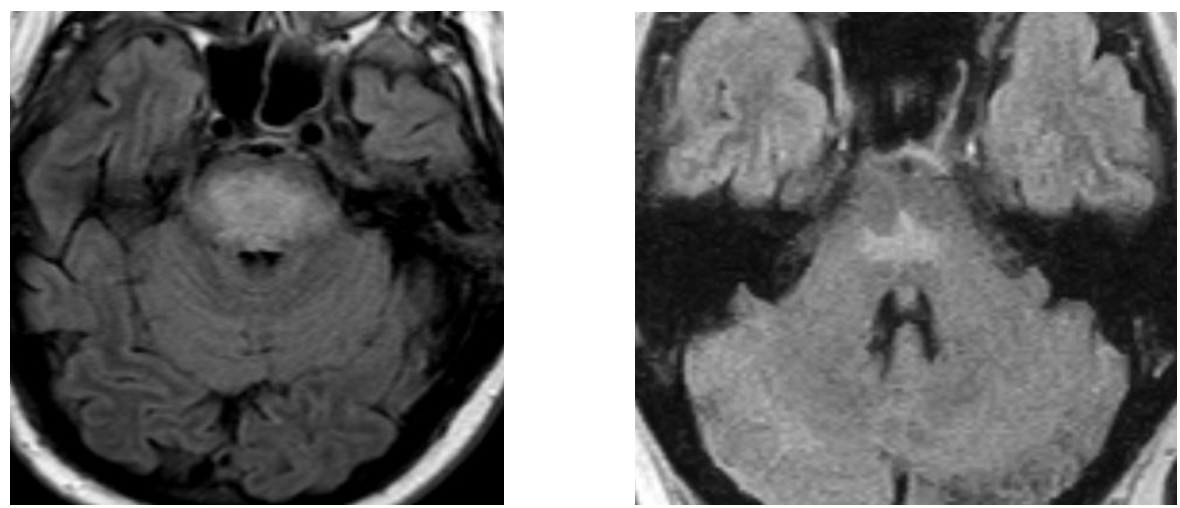

Figure 2. Follow-up MRI FLAIR sequences. Left-to-right: On Day 3 of IVIG treatment, there is increased hyperintensity in central pons, associated with clinical worsening; 6 months after IVIG treatment, there is decreased hyperintensity in central pons, with residual limited to the area of diffusion restriction on the initial MRI.

extremities. He reported being back to baseline in approximately 3 months.

On 6-month follow up, he had $5 / 5$ strength in all extremities. Repeat MRI revealed significant improvement in the pontine $\mathrm{T} 2 \mathrm{~W}$ hyperintensity, with remaining hyperintensity confined to the region of previous diffusion restriction (Figure 2B).

\section{DISCUSSION}

The diagnosis of CPM was confirmed by clinical history, risk factors, exam and MRI findings. Encephalopathy with pseudobulbar symptoms, and flaccid quadriparesis in the setting of alcoholism and more than $8 \mathrm{mmol} / \mathrm{L} /$ day correction of hyponatremia is a common presentation. ${ }^{1}$ Characteristic MRI in CPM is hyperintensity in the central pons and/or extrapontine structures (basal ganglia and thalamus) on T2W and FLAIR sequences, and hypointensity on T1W MRI. ${ }^{6}$ This patient's MRI fulfilled these criteria.
CPM has variable prognosis, and is potentially devastating. We used IVIG according to reported protocols $^{4}{ }_{5}$ despite the patient clinically and radiologically worsening during the first three days of treatment. Whether this was due to the use of IVIG or the underlying CPM pathogenesis is unknown. The course of CPM is still poorly defined. It is possible that the patient would have improved without treatment, but we maintain this is unlikely given his severe clinical picture, and his rapid and significant improvement after only one week of treatment with return to baseline in 6 months, which is exceptional. Another issue is the diffusion restriction on the initial MRI, which may herald poor prognosis. In extrapontine myelinolysis(EPM), normal diffusion imaging is associated with good prognosis. ${ }^{7}$ Our patient's clinical improvement indicates that significant diffusion restriction and early clinical worsening do not preclude giving IVIG. Early imaging abnormalities may reflect edema rather than irreversible demyelination, and severity of lesions on imaging do not always correlate with prognosis. ${ }^{8}$ More studies are needed to determine the prognostic value of DWI and the benefit of IVIG in CPM. 


\section{REFERENCES}

1. Martin RJ. Central pontine and extrapontine myelinolysis: the osmotic demyelination syndromes. J Neurol Neurosurg Psychiatry 2004 Sep;75 Suppl 3:iii22-iii28.

2. Musana AK, Yale SH. Central pontine myelinolysis: case series and review. Wis Med J 2005; 104:56e60.

3. Laubenberger J, Schneider B, Ansorge O, Götz F, Häussinger D, Volk B, Langer M. Central pontine myelinolysis: clinical presentation and radiologic findings. Eur Radiol. 1996;6(2):177-83.

4. Finsterer J. Engelmayer E. Trinka E, et al. Immunoglobulins are effective in pontine myelinolysis. Clin Neuropharmacol. 2000 MarApr;23(2):110-3.

5. Deleu D, Salim K, Mesraoua B, El Siddig A, Al Hail H, Hanssens Y. "Man-in-the-barrel" syndrome as delayed manifestation of extrapontine and central pontine myelinolysis: beneficial effect of intravenous immunoglobulin. J Neurol Sci. 2005 Oct 15;237(12):103-6.

6. Kallakatta RN, Radhakrishnan A, Fayaz RK, Unnikrishnan JP, Kesavadas C, Sarma SP. Clinical and functional outcome and factors predicting prognosis in osmotic demyelination syndrome (central pontine and/or extrapontine myelinolysis) in 25 patients. J Neurol Neurosurg Psychiatry. 2010 Sep 8.

7. Shin HW, Song D, Sohn YH. Normal diffusion-weighted MR imaging predicts a good prognosis in extrapontine myelinolysis-induced parkinsonism. Mov Disord. 2009 Aug 15;24(11):1701-3.

8. Kawahara I, Tokunaga Y, Ishizaka S, Yagi N. Reversible clinical and magnetic resonance imaging of central pontine myelinolysis following surgery for craniopharyngioma: serial magnetic resonance imaging studies. Neurol Med Chir (Tokyo). 2009 Mar;49(3):120-3. 